\title{
EPOS Multi-scale laboratories Data Services \& Trans-national access program
}

Geertje ter Maat, Richard Wessels, Otto Lange, Ernst Willingshofer, Aldo Winkler, Audrey Ougier-Simonin, Elisabetta Del Bello, Fabio Corbi, Francesca Funiciello, Kirsten Elger, Matthias Rosenau. Piergiorgio Scarlato, Jose-Luis Fernandez-Turiel and Martyn Drury

The Multi-scale Laboratories (MSL) represent a community of European solid Earth sciences laboratories and form one of the Thematic Cores Services (TCS) of the European Plate Observing System (EPOS), an ESFRI Landmark and European Research Infrastructure Consortium (ERIC). , . The diversity of experimental and analytical methods employed in such infrastructures reflects the multi-scale nature of the Earth system and is essential for the understanding of its evolution, for the assessment of geo-hazards, and for the sustainable exploitation of geo-resources.

The TCS MSL is committed to make Earth science laboratory data FAIR: Findable, Accessible, Interoperable, and Reusable. For this purpose, the TCS MSL has developed an online portal that brings together DOI-referenced data publications from research data repositories related to the TCS MSL context (https://epos-msl.uu.nl/).

In addition, the TCS MSL has developed a Trans-national access (TNA) program that allows researchers and research teams to apply for physical or remote access to the participating EPOS MSL laboratories. Access is currently supported by national funding or in-kind contribution. Based on the EPOS Data policy \& TNA General Principles, access is regulated by common rules and a transparent policy, including procedures and mechanisms for application, negotiation, proposal evaluation, user feedback, use of laboratory facilities and data curation.

Access to EPOS Multi-scale laboratories is a unique opportunity to create new synergy, collaboration and innovation, in a framework of trans-national access rules. Unaffiliated European solid Earth sciences laboratories are welcome and encouraged to join the growing TCS MSL community.

Words:244/250 